\title{
FREE VIBRATION OF AXIALLY FUNCTIONALLY GRADED EULER-BERNOULLI BEAMS
}

\author{
Stanisław Kukla, Jowita Rychlewska \\ Institute of Mathematics, Czestochowa University of Technology \\ Częstochowa, Poland \\ stanislaw.kukla@im.pcz.pl, jowita.rychlewska@im.pcz.pl
}

\begin{abstract}
In this contribution, free vibration of axially functionally graded beams is analysed within the framework of the Euler-Bernoulli beam theory. The beams with uniaxial variation of the elasticity modulus and mass density are approximated by an equivalent beam with piecewise exponentially varying geometrical and material properties. A numerical example for a beam with pinned ends is presented.
\end{abstract}

Keywords: axially functionally graded beams, free vibration, Euler-Bernoulli beam theory

\section{Introduction}

Functionally graded (FG) beams are composites characterized by the volume fraction of different materials (ceramic and metal) which is varied continuously with the thickness and/or the length of the beam. Through an appropriate selection of the volume fraction the FG beam with expected thermal and mechanical properties can be obtained. Therefore, the FG beams can be used in various engineering applications.

Vibration of non-homogenous beams is the subject of investigations presented in many papers. The free vibrations of FG beams was analyzed by using various methods in papers [1-5]. The finite element method to the free vibration problem of a FG beams was applied by Alshorbagy et al. in paper [1]. The presented analysis concerns the FG beams assuming a simple power law of a change of the material distribution through the thickness or the longitudinal direction. Hein and Feklistova [2] present an application of the Haar wavelet approach to free vibrations of FG beams with various boundary conditions and varying cross-sections. In paper [3] by Anandrao et al. the finite element system of equations to free vibration analysis of the FG beams is derived. The variation of material properties across the thickness of the beam was governed by a power law distribution. The free vibration and stability of tapered beams made of axially FG materials were studied by Shahba and Rajasekaran [4]. The solution to the problem was obtained by applying a differential transform element method. The exact solution to free vibration of axially exponentially graded beams is presented by Li et al. in reference [5]. Free vibration 
of axially FG beams consisting of two segments was studied in paper [6]. The analytical solution to the problem was obtained by assumption that the changes of the cross-sectional area and material properties in the beam segments have an exponential form.

This paper presents a solution to the problem of free vibration of a beam consisting of an arbitrary number of axially exponentially graded beam segments. The frequency equation is numerically solved. The eigenfrequencies are presented in a tabular form.

\section{Mathematical formulation of the problem}

Consider an axially graded and non-uniform beam of length $L$. It is assumed that material properties and/or cross-section of the beam vary continuously along the length direction. According to the Euler-Bernoulli beam theory, the governing differential equation is given by

$$
\frac{\partial^{2}}{\partial x^{2}}\left[E(x) I(x) \frac{\partial^{2} w}{\partial x^{2}}\right]+\rho(x) A(x) \frac{\partial^{2} w}{\partial t^{2}}=0, \quad 0<x<L,
$$

where $x$ is axial coordinate, $w(x, t)$ is the transverse deflection of the beam at the position $x$ and time $t, E(x)$ is the Young's modulus, $I(x)$ denotes the moment of inertia, $\rho(x)$ and $A(x)$ denote the mass density and cross-sectional area, respectively. In this contribution it is assumed that

$$
E(x) I(x)=D f(x) \quad \rho(x) A(x)=m f(x), \quad 0<x<L,
$$

where $D$ is a reference value of $E I$ at $x=0, m$ is a reference value of $\rho A$ at $x=0$ and $f$ is a function which has continuous derivatives up to the second order. The function $f(x)$ in intervals $x_{i-1}<x<x_{i}, i=1, . ., n$, is approximated by the functions of exponential form, i.e.

$$
f(x) \cong d_{i} e^{2 \beta_{i} \frac{x}{L}} \quad \text { for } x_{i-1}<x<x_{i}, \quad i=1, \ldots, n
$$

with $x_{0}=0$ and $x_{n}=L$. It is assumed that $f\left(x_{i}\right)=d_{i} e^{2 \beta_{i} \frac{x_{i}}{L}}, i=0,1, . ., n$. Taking into account equations (3) in equations (2), one obtains

$$
\begin{aligned}
& E(x) I(x) \cong D d_{i} e^{2 \beta_{i} \frac{x}{L}} \text { for } x_{i-1}<x<x_{i}, \quad i=1, \ldots, n \\
& \rho(x) A(x) \cong m d_{i} e^{2 \beta_{i} \frac{x}{L}}
\end{aligned}
$$


Denoting the length of the $i$-th element as $\Delta x_{i}=x_{i}-x_{i-1}$, the gradient parameters $\beta_{i}$ are given by

$$
\beta_{i}=\frac{L}{2 \Delta x_{i}} \ln \frac{f\left(x_{i}\right)}{f\left(x_{i-1}\right)}, \quad i=1, \ldots, n
$$

Assuming the transverse displacement of the beam in the form $w(x, t)=w_{i}(x, t)$ for $x_{i-1}<x<x_{i}, i=1, \ldots, n$, the governing equation for such a piecewise beam can be expressed by

$$
\frac{\partial^{2}}{\partial x^{2}}\left[D d_{i} e^{2 \beta_{i} \frac{x}{L}} \frac{\partial^{2} w_{i}}{\partial x^{2}}\right]+m d_{i} e^{2 \beta_{i} \frac{x}{L}} \frac{\partial^{2} w_{i}}{\partial t^{2}}=0, \quad x_{i-1}<x<x_{i}, \quad i=1, \ldots, n
$$

Setting

$$
w_{i}(x, t)=W_{i}(x) \sin \omega t, \quad i=1, \ldots, n,
$$

where $W_{i}(x), i=1, \ldots, n$, are the corresponding amplitude functions and $\omega$ is the circular frequency of the beam, one obtains

$$
\frac{d^{2}}{d x^{2}}\left[D e^{2 \beta_{i} \frac{x}{L}} \frac{d^{2} W_{i}}{d x^{2}}\right]-m \omega^{2} e^{2 \beta_{i} \frac{x}{L}} W_{i}=0, \quad x_{i-1}<x<x_{i}, \quad i=1, \ldots, n
$$

Introducing the non-dimensional variables

$$
\xi=\frac{x}{L}, \quad \quad \Omega^{4}=\frac{m}{D} L^{4} \omega^{2}
$$

equations (8) can be rewritten as

$$
\frac{d^{2}}{d \xi^{2}}\left[e^{2 \beta_{i} \xi} \frac{d^{2} W_{i}}{d \xi^{2}}\right]-\Omega^{4} e^{2 \beta_{i} \xi} W_{i}=0, \quad \xi_{i-1}<\xi<\xi_{i}, \quad i=1, \ldots, n
$$

After some transformations we can rewrite equations (10) as follows:

$$
\frac{d^{4} W_{i}}{d \xi^{4}}+4 \beta_{i} \frac{d^{3} W_{i}}{d \xi^{3}}+4 \beta_{i}^{2} \frac{d^{2} W_{i}}{d \xi^{2}}-\Omega^{4} W_{i}=0, \quad \xi_{i-1}<\xi<\xi_{i}, \quad i=1, \ldots, n
$$

\section{Solution of the free vibration problem}

The general solution of equations (11) has the following form

$$
W_{i}(\xi)=e^{-\beta_{i} \xi}\left(A_{i} \cos \delta_{i} \xi+B_{i} \sin \delta_{i} \xi+C_{i} \cosh \bar{\delta}_{i} \xi+D_{i} \sinh \bar{\delta}_{i} \xi\right), \quad \xi_{i-1}<\xi<\xi_{i},
$$

where $\delta_{i}=\sqrt{\Omega^{2}-\beta_{i}^{2}}, \bar{\delta}_{i}=\sqrt{\Omega^{2}+\beta_{i}^{2}}, i=1, \ldots, n$. 
The above solutions will be applied to boundary conditions for pinned-pinned beam as follows

$$
W_{1}(0)=W_{n}(1)=0 \quad \frac{d^{2} W_{1}}{d \xi^{2}}(0)=\frac{d^{2} W_{n}}{d \xi^{2}}(1)=0
$$

The matching conditions between two connecting elements of the piecewise beams satisfy the following continuity conditions

$$
\begin{aligned}
& W_{i}\left(\xi_{i}\right)=W_{i+1}\left(\xi_{i}\right) \quad \frac{d W_{i}}{d \xi}\left(\xi_{i}\right)=\frac{d W_{i+1}}{d \xi}\left(\xi_{i}\right) \\
& \frac{d^{2} W_{i}}{d \xi^{2}}\left(\xi_{i}\right)=\frac{d^{2} W_{i+1}}{d \xi^{2}}\left(\xi_{i}\right) \quad \frac{d^{3} W_{i}}{d \xi^{3}}\left(\xi_{i}\right)=\frac{d^{3} W_{i+1}}{d \xi^{3}}\left(\xi_{i}\right), \quad i=1, \ldots, n-1
\end{aligned}
$$

Substituting functions (12) into boundary and continuity conditions given by equations (13) and (14), we obtain the free vibration problem in the form

$$
\mathbf{A}(\omega) \cdot \mathbf{X}=\mathbf{0}
$$

where $\mathbf{X}=\left[A_{1}, B_{1}, C_{1}, D_{1}, \ldots, A_{n}, B_{n}, C_{n}, D_{n}\right]^{T}$ and $\mathbf{A}(\omega)=\left[a_{k j}\right]_{4 n \times 4 n}$. Two first rows of the matrix $\mathbf{A}$ represent the boundary conditions at $\xi=0$ and two last rows of $\mathbf{A}$ represent the boundary conditions at $\xi=1$. The rows of the matrix A with nonzero elements $a_{4 i-1,4 i-3}, \ldots, a_{4 i-1,4 i+4}, a_{4 i, 4 i-3}, \ldots, a_{4 i, 4 i+4}, a_{4 i+1,4 i-3}, \ldots, a_{4 i+1,4 i+4}$, $a_{4 i+2,4 i-3}, \ldots, a_{4 i+2,4 i+4}, i=1, \ldots, n-1$, are determined by the continuity conditions at $\xi=\xi_{i}, i=1, \ldots, n-1$. The determinant of the matrix $\mathbf{A}$ has to vanish for a nontrivial solution of the frequency equation of the beam under consideration to exist. Frequency equation

$$
\operatorname{det} \mathbf{A}(\omega)=0
$$

is then solved numerically using an approximate method.

\section{Numerical example}

The numerical computations were performed for pinned-pinned FG beam with $n$ segments of the same length. The function $f($.$) occurring in equations$ (2), (3) is assumed in one of the forms: $f(\xi)=1+\xi^{p}$ for $p=0.5 ; 1.0 ; 2.0$, or $f(\xi)=1+0.5 \sin (a \pi \xi)$ for $a=1.0 ; 2.0 ; 3.0$, where $\xi=x / L$. The calculations were carried out for various numbers of segments $n=2 ; 5 ; 10 ; 15$. 
Four dimensionless eigenfrequencies of the FG beam with $\boldsymbol{n}$ segments of constant length with $f(\xi)=1+\xi^{p}$

\begin{tabular}{|c|c|c|c|c|}
\hline \multirow{3}{*}{0.5} & 2 & 5 & 10 & 15 \\
\hline \multirow{4}{*}{1} & 3.001006 & 3.010238 & 3.036439 & 3.045427 \\
\cline { 2 - 5 } & 6.285830 & 6.173596 & 6.187237 & 6.198689 \\
\cline { 2 - 5 } & 9.388662 & 9.354648 & 9.335179 & 9.344616 \\
\cline { 2 - 5 } & 12.570167 & 12.538667 & 12.486328 & 12.489851 \\
\hline \multirow{4}{*}{1} & 3.095507 & 3.096357 & 3.096695 & 3.096761 \\
\cline { 2 - 5 } & 6.286651 & 6.266571 & 6.266667 & 6.266697 \\
\cline { 2 - 5 } & 9.415975 & 9.415099 & 9.415025 & 9.415040 \\
\cline { 2 - 5 } & 12.569686 & 12.560122 & 12.559585 & 12.559592 \\
\hline \multirow{3}{*}{2} & 3.207782 & 3.207477 & 3.207418 & 3.207406 \\
\cline { 2 - 5 } & 6.286086 & 6.326810 & 6.327096 & 6.327154 \\
\cline { 2 - 5 } & 9.454665 & 9.455613 & 9.455755 & 9.455796 \\
\cline { 2 - 5 } & 12.569836 & 12.589830 & 12.590206 & 12.590236 \\
\hline
\end{tabular}

Table 2

Four dimensionless eigenfrequencies of the FG beam with $\boldsymbol{n}$ segments of constant length with $f(\xi)=1+0.5 \sin (a \pi \xi)$

\begin{tabular}{|c|c|c|c|c|}
\hline \multirow{3}{*}{$a$} & 2 & 5 & 10 & 15 \\
\hline \multirow{4}{*}{1} & 2.810172 & 2.836856 & 2.838765 & 2.839073 \\
\cline { 2 - 5 } & 6.278107 & 6.145092 & 6.145823 & 6.145838 \\
\cline { 2 - 5 } & 9.339187 & 9.337856 & 9.339502 & 9.339522 \\
\cline { 2 - 5 } & 12.568765 & 12.501314 & 12.504811 & 12.504871 \\
\hline \multirow{4}{*}{2} & 3.141592 & 2.654107 & 2.602609 & 2.593108 \\
\cline { 2 - 6 } & 6.283185 & 6.393857 & 6.475257 & 6.491527 \\
\cline { 2 - 6 } & 9.424777 & 9.451843 & 9.422380 & 9.427523 \\
\cline { 2 - 6 } & 12.566370 & 12.575262 & 12.555303 & 12.553347 \\
\hline \multirow{3}{*}{3} & 3.460695 & 2.806813 & 2.699744 & 2.680683 \\
\cline { 2 - 6 } & 6.268350 & 5.128893 & 4.988445 & 4.948780 \\
\cline { 2 - 6 } & 9.573864 & 9.012672 & 9.379156 & 9.428255 \\
\cline { 2 - 6 } & 12.573379 & 12.668699 & 12.271558 & 12.255414 \\
\hline
\end{tabular}

Four dimensionless eigenfrequencies for the function $f(\xi)=1+\xi^{p}$ are presented in Table 1 and for the function $f(\xi)=1+0.5 \sin (a \pi \xi)$ are shown in Table 2 . 
From Table 1 it is seen that the obtained results for $n=5$ and $n=10$ differ at most by $0.87 \%$ and for $n=10$ and $n=15$ they differ at most by $0.29 \%$. Analogous comparison from Table 2 gives the differences of the results 4.07 and $0.79 \%$, respectively.

\section{Conclusions}

The main conclusion of this contribution is that the proposed approach can be applied to the analysis of free vibration problems for axially functionally graded beams. The primary idea presented here is to approximate the FG beam by the beam with piecewise exponentially varying geometrical and material properties. The example shows that the accuracy of the numerically obtained eigenfrequencies improves as the number of segments increases. The numerical example presented in this paper concerns a pinned-pinned beam, but the proposed method may be used in the vibration analysis of FG beams with other boundary conditions.

\section{References}

[1] Alshorbagy A.E., Eltaher M.A., Mahmoud F.F., Free vibration characteristics of a functionally graded beam by finite element method, Appl. Math. Model. 2011, 35, 412-425.

[2] Hein H., Feklistova L., Free vibrations of non-uniform and axially functionally graded beams using Haar wavelets, Eng. Struct. 2011, 33, 3696-3701.

[3] Anandrao K.S., Gupta R.K., Ramachandran P., Venkateswara Rao G., Free vibration analysis of functionally graded beams, Def. Sci. J. 2012, 62(3), 139-146.

[4] Shahba A., Rajasekaran S., Free vibration and stability of tapered Euler-Bernoulli beams made of axially functionally graded materials, Appl. Math. Model. 2012, 36, 3094-3111.

[5] Li X.-F., Kang Y.-A., Wu J.-X., Exact frequency equations of free vibration of exponentially functionally graded beams, Appl. Acoustic 2013, 74, 413-420.

[6] Kukla S., Rychlewska J., Free vibration analysis of functionally graded beams, J. Appl. Math. Comp. Mech. 2013, 12(2), 39-44. 